\title{
Magnetomechanical performance of directionally solidified Fe-Ga alloys
}

\author{
X. Zhao, ${ }^{\text {a) }}$ N. Mellors, and D. G. Lord \\ Institute for Materials Research, University of Salford, Salford M5 4WT, United Kingdom
}

(Presented on 9 January 2007; received 30 October 2006; accepted 13 December 2006; published online 2 May 2007)

\begin{abstract}
Iron-gallium alloys can produce magnetostrictions of $\sim 400 \mathrm{ppm}$ and might serve as mechanically robust actuator/sensing materials. However, for polycrystalline $\mathrm{Fe}-\mathrm{Ga}$ alloys, the magnetostrictive performance decreases with the increasing deviations from the ideal $\langle 100\rangle$ texture. In this paper, three directionally solidified $\mathrm{Fe}-\mathrm{Ga}$ alloys with gallium contents of $17,18.4$, and 19.5 at. \% were characterized at ambient temperature. These specimens exhibit high $d_{33}$ and magnetic permeability when subjected to applied magnetic fields, indicating their suitability for light weight actuator applications but not for high force applications due to their low saturation magnetostriction and hence low blocking force. All the alloys produce significant changes in magnetization, around $0.7 M_{s}-0.8 M_{s}$ when subjected to cyclic compressive stresses of $51 \mathrm{MPa}$, making them promising candidate materials for sensing and energy harvesting applications. However, eddy current effects may easily become a problem when such materials are subjected to a high frequency vibration or magnetic field due to their intrinsic high magnetic permeability. (c) 2007 American Institute of Physics. [DOI: 10.1063/1.2711701]
\end{abstract}

\section{INTRODUCTION}

Magnetostrictive materials, such as Terfenol-D, can be used not only as actuator materials but also as sensing or energy harvesting materials. ${ }^{1,2}$ However, the intrinsic brittleness of Terfenol-D, especially under tensile stresses, puts certain limitations on the design for all types of applications.

Alloys of iron substituted with nonmagnetic gallium also exhibit coupling between their mechanical and magnetic states and offer promise as mechanically robust actuator/ sensing materials. ${ }^{3}$ Studies of single crystal $\mathrm{Fe}_{100-x} \mathrm{Ga}_{x}$, where $13<x<23$, have shown that the alloys exhibit magnetostrictive strains approaching $400 \mathrm{ppm}$, with low saturating fields of several hundred oesteds, as well as displaying a limited temperature dependence over a $-20-80{ }^{\circ} \mathrm{C}$ range. ${ }^{4-6}$ However, for polycrystalline $\mathrm{Fe}-\mathrm{Ga}$ alloys, the magnetostrictive performance decreases with the increasing deviations from the ideal $\langle 100\rangle$ texture. $^{7}$ How to obtain a preferred crystallographic texture and choose suitable postgrowth processing procedures to optimise the magnetostrictive performance still remains a challenge. In this paper, three directionally solidified $\mathrm{Fe}-\mathrm{Ga}$ alloy specimens have been characterized and their suitability for magnetostrictive actuator/sensing or energy harvesting applications is discussed.

\section{EXPERIMENT}

All three $\mathrm{Fe}-\mathrm{Ga}$ samples were supplied by ETREMA Products, Inc. These specimens were prepared by a freestanding zone melting (FSZM) technique resulting in highly textured specimens, with a strong $\langle 100\rangle$ fiber texture. They were all machined to $6.35 \mathrm{~mm}$ in diameter and $50 \mathrm{~mm}$ in length and contained (A) 17 at. \%, (B) 18.4 at. \%, and (C) 19.5 at. $\%$ nominal gallium contents.

${ }^{a)}$ Electronic mail: x.zhao@salford.ac.uk
To achieve uniform magnetic fields and high stresses in the characterization, an electromagnet with a modified pole piece was used. A cylindrical portion of one pole face was removed and remounted with $\mathrm{O}$ rings and then filled with compressed air to supply the pressure. The changes in magnetic induction were measured by a flux meter with a compensated pickup coil wound around the sample, while the changes in longitudinal strain were measured with a strain gauge positioned at the midlength of the rod. The strength of magnetic field was measured with a Hall probe positioned close to the strain gauge.

Specimens were measured in as-received condition and all measurements were carried out at ambient temperature.

\section{RESULTS AND DISCUSSION}

Magnetostriction and magnetization loops against applied magnetic field were obtained at constant applied compressive stresses, the stresses ranging from 0 up to $51 \mathrm{MPa}$ with a step size of $3 \mathrm{MPa}$. From Fig. 1, it can be seen that $\mathrm{Fe}_{83} \mathrm{Ga}_{17}$ shows the lowest saturating field, the smallest hysteresis, and the highest $d_{33}$, the changing rate of magnetostriction against field. An unusual magnetization process can be seen around zero field when the rod had no stress applied in that the magnetostriction decreased with the increase of magnetic field in some ranges; this behavior was confirmed by the step around zero field in the hysteresis loop. $\mathrm{Fe}_{81.6} \mathrm{Ga}_{18.4}$ exhibited a strain-jump effect when a compressive stress was applied, similar to that obtained from twinned Terfenol-D, and the jump shifted to higher fields when the applied stress was increased.

Figure 1 also shows, plotted against applied compressive stress, the strain at zero applied field and the achievable maximum magnetostriction. The two curves cross each other at one point where the corresponding stress is the so-called blocking stress, above which no further magnetostrictive dis- 

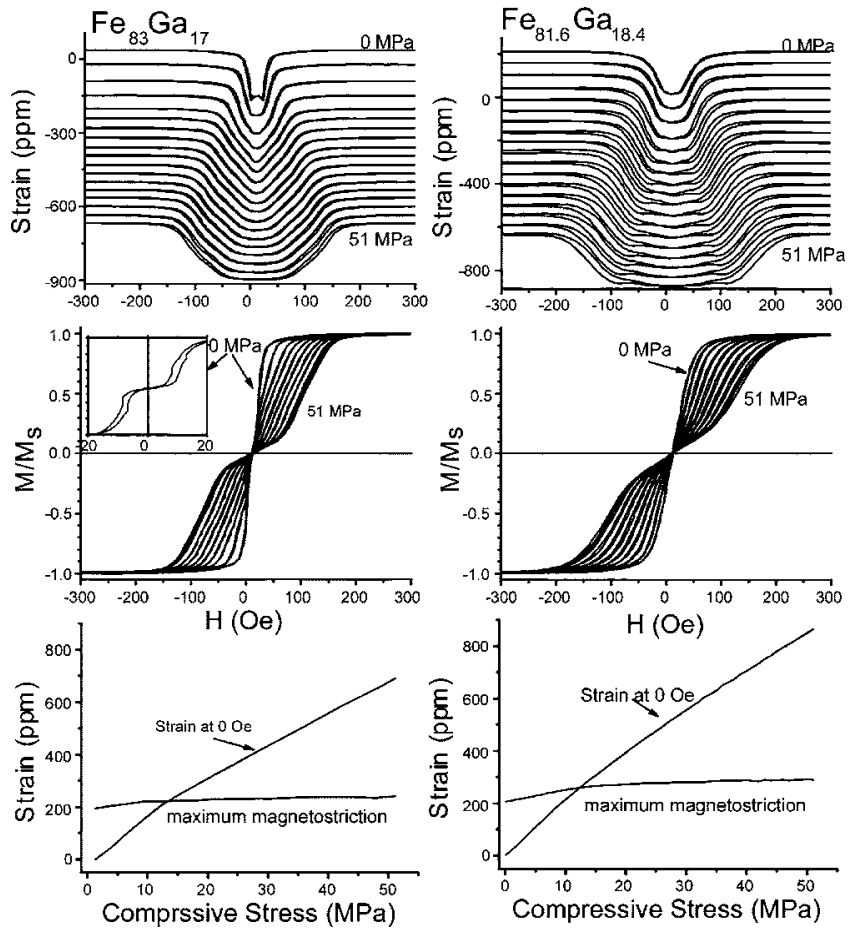

FIG. 1. Magnetostriction and magnetization loops measured at constant applied compressive stresses are plotted against applied magnetic fields, the stresses ranging from 0 up to $51 \mathrm{MPa}$ with an incremental step size of $3 \mathrm{MPa}$ used for magnetostriction loops but $6 \mathrm{MPa}$ for magnetization loops and strain-magnetization loops. Also plotted are the strains measured at 0 Oe and maximum achievable magnetostrictions when prestresses were changed.

placement can be produced. This has values of around $12 \mathrm{MPa}$ for $\mathrm{Fe}_{83} \mathrm{Ga}_{17}$ and $\mathrm{Fe}_{81.6} \mathrm{Ga}_{18.4}$ but only $7 \mathrm{MPa}$ for $\mathrm{Fe}_{80.5} \mathrm{Ga}_{19.5}$ (not shown in Fig. 1) when no prestress is present. The blocking stress can be increased when a prestress is applied to the sample; however, the values found are still much lower in FeGa alloys than those found in Terfenol-D: this is because of lower Young's modulus and even lower saturated magnetostriction.

The saturation magnetostriction of the Fe-Ga samples lies in the range of 200-300 ppm. Hard Young's modulus, calculated from the strain-stress curves, decreases with the increase in the gallium content from $80 \mathrm{GPa}$ for 17 at. $\% \mathrm{Ga}$ to $54 \mathrm{GPa}$ for 19.5 at. $\% \mathrm{Ga}$. By contrast, the commercially available Bridgman grown Terfenol-D has a corresponding value of $90 \mathrm{GPa}$ in hard Young's modulus and over 1800 ppm in saturation magnetostriction: its blocking stress can reach up to $100 \mathrm{MPa}$. These Fe-Ga alloys might be more suitable for lightweight actuator applications due to their high $d_{33}$ values and low saturating fields.

In addition to being usually used as actuator materials, "giant" magnetostrictive materials can also serve as sensing or energy harvesting materials for converting mechanical vibrations into voltage signals or electric power. In such applications the dependence of magnetization change upon the applied stress/strain needs to be investigated.

Partly shown in Fig. 2, the major loops of magnetization against applied stress have been directly measured using applied cyclic compressive stresses up to $25 \mathrm{MPa}$ at different constant magnetic bias fields. It can be seen that $\mathrm{Fe}_{83} \mathrm{Ga}_{17}$ is
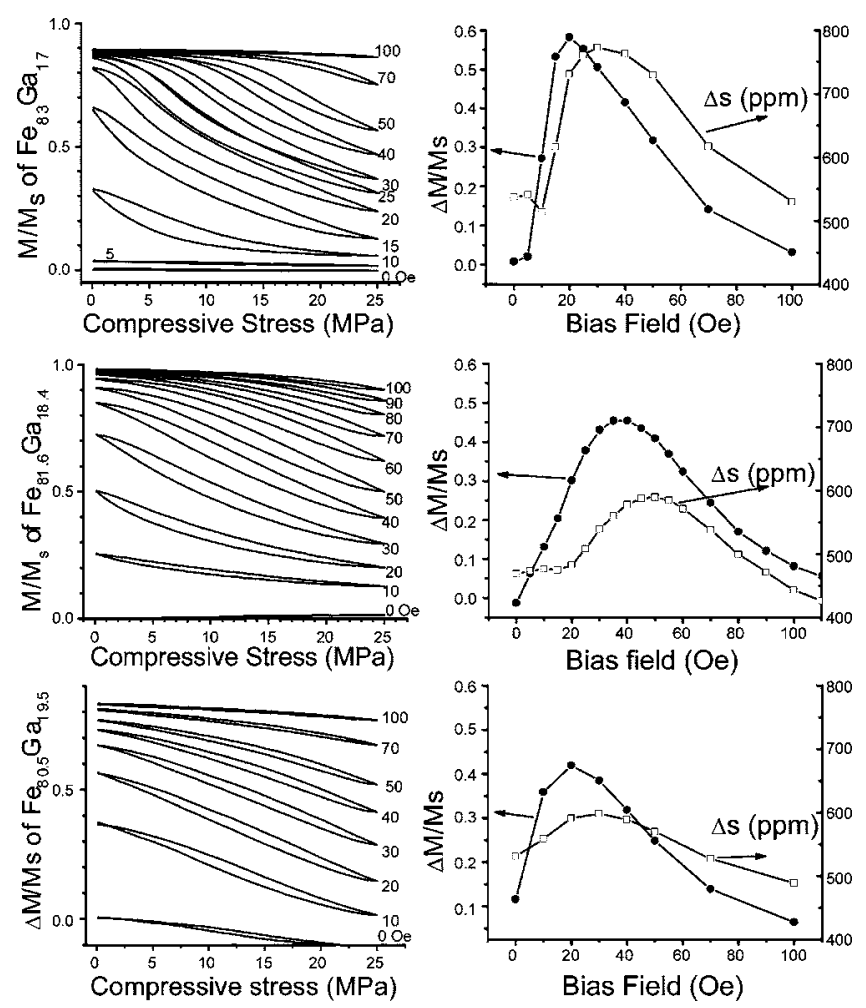

FIG. 2. Major loops of magnetization obtained with applied cyclic compressive stresses up to $25 \mathrm{MPa}$, at different constant magnetic bias fields, are plotted. The total changes in magnetization and strain, namely, $\Delta M$ and $\Delta s$, are also plotted against applied bias field.

more sensitive to applied stresses than the other samples, although the change in magnetization $\Delta M$ with stress is more linear for $\mathrm{Fe}_{81.6} \mathrm{Ga}_{18.4}$ and $\mathrm{Fe}_{80.5} \mathrm{Ga}_{19.5}$ than for $\mathrm{Fe}_{83} \mathrm{Ga}_{17}$. The $\Delta M$ for a cyclic stress of $25 \mathrm{MPa}$ can reach up to $0.6 M_{s}$ for $\mathrm{Fe}_{83} \mathrm{Ga}_{17}$, with a bias field of $20 \mathrm{Oe}$, while for $\mathrm{Fe}_{80.5} \mathrm{Ga}_{19.5}$, the corresponding value is only $0.4 M_{s}$. $\mathrm{Fe}_{81.6} \mathrm{Ga}_{18.4}$ has a $\Delta M$ of $0.4 M_{s}$, but to achieve this value requires a higher bias field of 40 Oe. It should be noted that for all three samples, the bias field corresponding to the maximum change in strain is always about 10 Oe higher than that for the maximum value of $\Delta M$.

Figure 3 shows the major loops of magnetization measured with applied cyclic compressive stresses up to $50 \mathrm{MPa}$ at different constant magnetic bias fields. With the increase of the maximum applied stress, $\mathrm{Fe}_{83} \mathrm{Ga}_{17}$ shows a much more linear relation between $\Delta M$ and applied stress and it still produces the highest $\Delta M$ value, now of $0.8 M_{s}$. For $\mathrm{Fe}_{81.6} \mathrm{Ga}_{18.4}$ the value of $\Delta M$ becomes $0.7 M_{s}$ and, though still lower than that from $\mathrm{Fe}_{83} \mathrm{Ga}_{17}$, the difference between $\mathrm{Fe}_{83} \mathrm{Ga}_{17}$ and $\mathrm{Fe}_{81.6} \mathrm{Ga}_{18.4}$ is less significant than that with stress up to only $25 \mathrm{MPa}$.

To try to obtain a clearer idea about the magnetization processes, the loops of magnetization against strain are plotted in Fig. 3. For $\mathrm{Fe}_{83} \mathrm{Ga}_{17}$, with the bias field ranging from 15 to $90 \mathrm{Oe}$, the magnetization changes linearly with strain in most regions. By subtracting the mechanical contribution from the measured strains, further calculations show that the changing rate contributed by magnetostrictive processes is about $1 M_{s} / \lambda_{s}$, where $\lambda_{s}$ is the saturation magnetostriction ( 240 ppm for $\mathrm{Fe}_{83} \mathrm{Ga}_{17}$ ), indicating a simple magnetization 

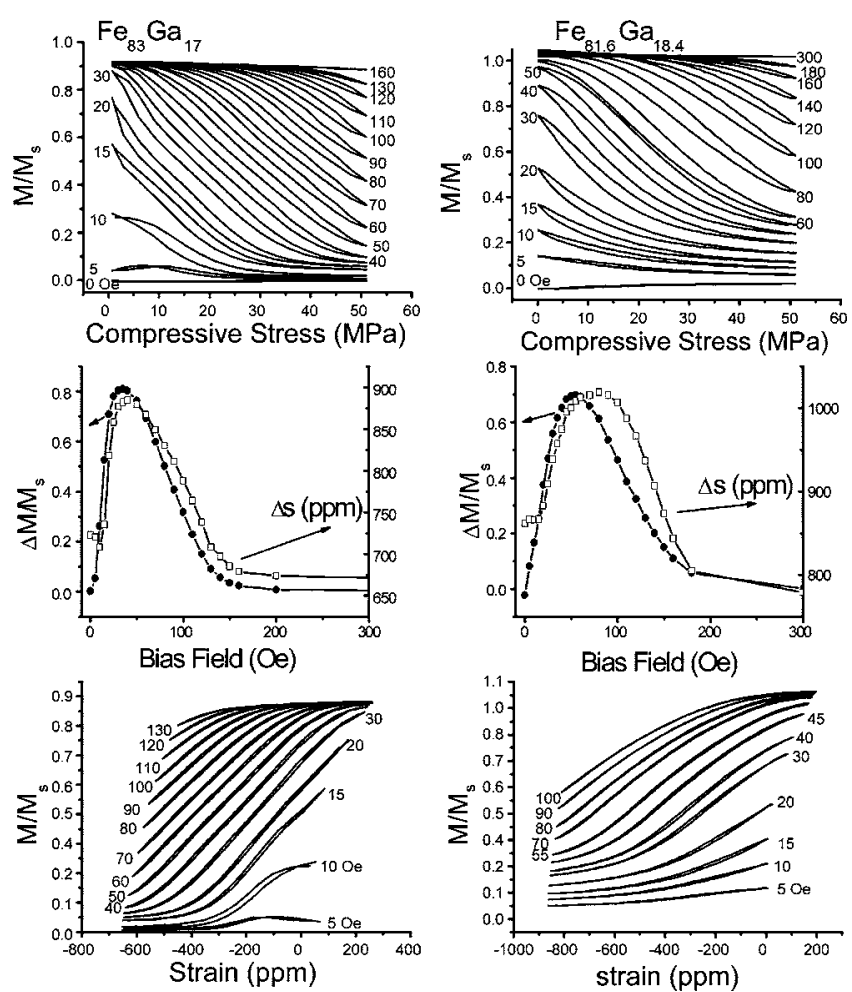

FIG. 3. Major loops of magnetization obtained with applied cyclic compressive stresses up to $51 \mathrm{MPa}$ at different constant magnetic bias fields are plotted. The total changes in magnetization and strain, namely, $\Delta M$ and $\Delta s$, against applied bias field and the magnetizations against strains are also plotted.

process that is most likely domain wall movement. However, for $\mathrm{Fe}_{81.6} \mathrm{Ga}_{18.4}$, the magnetization process appears to be more complicated.

In general, higher $\Delta M$ values usually indicate that a material has a higher capability of harvesting more energy from a given amplitude mechanical vibration. Galfenol has been found to give a maximum change in magnetization of $0.8 M_{s}$, higher than the $0.6 M_{s}$ commonly obtained with Terfenol-D (Ref. 8) when subjected to a cyclic stress of $50 \mathrm{MPa}$. Also, Galfenol with 17 at. \% gallium has a saturated magnetization of $1400 \mathrm{kA} / \mathrm{m}(1.75 \mathrm{~T})$, much higher than the $1 \mathrm{~T}$ Terfenol-D value. Both of these features would seem to indicate that Galfenol was a good candidate for energy harvesting applications. However, Galfenol has a very high magnetic permeability relative to Terfenol, and this makes the eddy current effect much more significant when the magne- tization is changed either by external fields or by applied stresses and measures such as lamination would have to be chosen to reduce this effect. ${ }^{2}$

\section{CONCLUSIONS}

Three directionally solidified $\mathrm{Fe}-\mathrm{Ga}$ alloys with gallium content of (A) 17 at. \%, (B) 18.4 at. \%, and (C) 19.5 at. \% were characterized at ambient temperature. These specimens exhibit high $d_{33}$ and magnetic permeability when subjected to applied magnetic fields, which indicates that such materials are more suitable for light weight actuator applications but might not for high force applications due to their low saturated magnetostriction and Young's modulus and hence low blocking force. When subjected to cyclic compressive stresses, these specimens produce significant changes in magnetization, around $0.7 M_{s}-0.8 M_{s}$ for a stress of $51 \mathrm{MPa}$, much higher than that available from Terfenol-D. This makes such $\mathrm{Fe}-\mathrm{Ga}$ alloys promising candidate materials for sensing and energy harvesting applications. However, eddy current effects may become a problem when such materials are subjected to a high frequency vibration or magnetic field due to their intrinsic high magnetic permeability, and measures such as lamination have to be taken to reduce this effect.

\section{ACKNOWLEDGMENTS}

This work was generated in the context of the MESEMA project, funded under the Sixth Framework Programme of the European Community (Contract No. AST3-CT-2003502915). The authors would like to thank ETREMA Products, Inc. for supplying the samples and for the constructive comments from Eric Summers.

${ }^{1}$ G. Engdahl, Handbook of Giant Magnetostrictive Materials (Academic, New York, 2000).

${ }^{2}$ X. Zhao and D. G. Lord, J. Appl. Phys. 99, 08 M703 (2006).

${ }^{3}$ A. E. Clark, J. B. Restorff, M. Wun-Fogle, T. A. Lograsso, and D. L. Schlagel, IEEE Trans. Magn. 36, 3238 (2000).

${ }^{4}$ J. R. Cullen, A. E. Clark, M. Wun-Fogle, J. B. Restorff, and T. A. Lograsso, J. Magn. Magn. Mater. 226, 948 (2001).

${ }^{5}$ R. A. Kellogg, A. B. Flatau, A. E. Clark, M. Wun-Fogle, and T. A. Lograsso, J. Appl. Phys. 91, 7821 (2002).

${ }^{6}$ A. E. Clark, J. B. Restorff, M. Wun-Fogle, and T. A. Lograsso, Mater. Trans., JIM 43, 881 (2002).

${ }^{7}$ R. A. Kellogg, A. B. Flatau, A. E. Clark, M. Wun-Fogle, and T. A. Lograsso, Proceedings of ASME International Mechanical Engineering Congress and Exposition 2003, IMECE2003-43140, Washington, D. C., 15-21 November, 2003 (unpublished).

${ }^{8}$ X. Zhao and D. G. Lord (unpublished). 